\title{
Educación financiera como modelo de inclusión financiera para empoderamiento de grupos vulnerables
}

\section{Financial education as a model of financial inclusion for the empowerment of vulnerable groups}

\author{
CASTILLO-FLORES, Esther Sarai $\uparrow^{*} \&$ DE LA CUESTA-SOLIS, Edgar \\ Universidad Tecnológica de Altamira, Boulevard de los Ríos km 3+100, Puerto Industrial, Altamira, Tam. \\ ID $1^{\mathrm{er}}$ Autor: Esther Sarai, Castillo-Flores / ORC ID: 0000-0001-9335-872X, CVU CONACYT ID: 466727 \\ ID $1^{\text {er }}$ Coautor: Edgar, De La Cuesta-Solis / ORC ID: 000-0003-0246-2041
}

DOI: $10.35429 / J B E .2019 .8 .3 .27 .30$

Recibido 10 de Marzo, 2019; Aceptado 30 de Junio, 2019

\section{Resumen}

La presente investigación tiene como objetivo evaluar la educación financiera como mecanismo de inclusión al sector financiero a través de una prueba piloto que permita desarrollar un instrumento de medición para evaluar la competencia de la educación financiera en la población, los resultados obtenidos a través de la prueba piloto arrojaron que no existe diferencia por género en cuanto a las educación financiera o la falta de esta, permitió conocer que la educación financiera que se tiene es muy básica ya que solo el 50,2\% maneja la banca de primer piso y de estos $33 \%$ desconoce si el uso eficiente que se le da a estos servicios es eficiente. También se encontraron resultados que muestra que solo el 50\% maneja un fondo de inversión o de ahorro para el futuro pero de estos el 35\% confundió el sistema de ahorro y pensión que hacen por nómina. Esta prueba permite principalmente desarrollar un instrumento de evaluación en la educación financiera y además contribuye a realizar un análisis de los programas educativos y evaluar cómo implementar la educación financiera en los programas educativos a nivel básico escolar.

Inclusión Financiera, Educación Financiera, Empoderamiento

\begin{abstract}
The purpose of this research is to evaluate financial education as a mechanism for inclusion in the financial sector through a pilot test that allows the development of a measuring instrument to assess the competence of financial education in the population, the results obtained through the test pilot showed that there is no gender difference in terms of financial education or lack thereof, allowed to know that the financial education is very basic since only $50.2 \%$ manages the first floor banking and of these $33 \%$ do not know if the efficient use of these services is efficient. Results were also found showing that only $50 \%$ manage an investment or savings fund for the future but of these, 35\% confused the payroll and savings system. This test mainly allows the development of an evaluation instrument in financial education and also contributes to an analysis of educational programs and to evaluate how to implement financial education in educational programs at the basic school level.
\end{abstract}

Financial Inclusion, Financial Education, Empowerment

Citación: CASTILLO-FLORES, Esther Sarai \& DE LA CUESTA-SOLIS, Edgar. Educación financiera como modelo de inclusión financiera para empoderamiento de grupos vulnerables. Revista de Educación Básica. 2019, 3-8: 27-30.

\footnotetext{
*Correspondencia al Autor (Correo Electrónico: sflores@utaltamira.edu.mx)
}

$\dagger$ Investigador contribuyendo como primer autor. 


\section{Introducción}

La inclusión financiera es una herramienta que promueve un incremento en el bienestar y el mejoramiento de la calidad de vida de las personas, mediante la utilización de servicios financieros que promueven el ahorro, incremento de patrimonio y la creación de un fondo de ahorro para la vejez (Raccanello \& Herrera Guzmán, Educación e inclusión financiera , 2014).

Según estudios realizados en América Latina, las estimaciones indican que el ingreso y la educación tienen una correlación positiva con la probabilidad de tener crédito formal, lo que se traduce en la utilización de los servicios formales de la Banca y de acuerdo a este análisis se observa que el ingreso, el destino de los créditos, la educación dan como resultado la posibilidad de evitar el endeudamiento excesivo. (Tribín-Uribe, 2016).

Se ha demostrado que la educación financiera proporciona las herramientas necesarias que desarrollan habilidades que facilita la toma de decisiones eficientes en el uso de las finanzas personales, disminuyendo los gastos innecesarios y promoviendo el ahorro. La Organización para la Cooperación y el Desarrollo Económicos (OCDE) propone que la educación financiera forme parte de la currícula de los estudiantes desde niveles iniciales, en México corresponde principalmente al nivel básico de Primaria y Secundaria.

La educación que reciben los niños y jóvenes será lo que marcará su desarrollo profesional, sin embargo todos ellos tendrán la necesidad de saber utilizar sus finanzas tanto en lo personal como en su desarrollo profesional.

Saber manejar las finanzas desde temprana edad provee una mejor preparación en este sentido, lo que ayuda a afrontar mejor una crisis económica y genera bienestar social.

La educación financiera es importante porque a niños y jóvenes desde corta edad se les dota de los mecanismos para los que enfrentarse a las crisis económicas.

\section{Inclusión Financiera}

Dentro de las definiciones de Inclusión Financiera que son aceptadas universalmente, consideran a esta como un proceso que promueve un acceso accesible, formal de una extensa gama de productos y servicios financieros regulados, ampliando su uso a diversos segmentos de la sociedad sin importar el ingreso o clase, mediante la utilización de herramientas innovadoras y tecnológicas, así como la utilización de recursos que promuevan la educación, la sensibilización y otorgue herramientas para el uso eficiente de estos servicios financieros con el objetivo de promover el bienestar financiero y la inclusión económica y social, lo que a su vez genera mayores condiciones de estabilidad económica $\mathrm{y}$ disminuye el riesgo de crisis financieras. (Sarmiento, 2016)

Según la Comisión Nacional Bancaria y de Valores (CNBV) define la inclusión financiera como el acceso y uso de servicios financieros formales bajo una regulación apropiada que garantice esquemas de protección al consumidor y promueva la educación financiera para mejorar las capacidades financieras de todos los segmentos de la población. Dicha definición señala una visión multidimensional de la inclusión financiera, lo que destaca cuatro componentes fundamentales que son: Acceso, Uso, Protección y Educación Financiera. Señala como acceso financiero a la accesibilidad que tiene la población de acceder del sistema financiero, en cuanto a infraestructura y capacidad para ofrecer servicios productos financieros por parte de las instituciones financiera como institución que oferta el servicio, como uso se refiere a la demanda de productos $y$ servicios financieros por la población, así como la frecuencia con la que los manejan ya que esto refleja el comportamiento y las necesidades de la población. Como Protección y defensa al consumidor se refiere a la regulación y los mecanismos que garantizan la transparencia para atender quejas de los consumidores y de las malas prácticas por parte de las instituciones financieras, así como el resguardo de los datos de los usuarios. 
Por ultimo hace referencia a la educación financiera para que la población adquiera aptitudes, conocimientos y habilidades para evaluar, analizar y tomar decisiones eligiendo productos y servicios financieros que garanticen el uso eficiente de las finanzas personales con el objetivos de mejorar el bienestar económico personal y de las familias (Consejo Nacional de Inclusion Financiera, 2018).

\section{Educación Financiera}

Según datos de la Organización para la Cooperación y el Desarrollo Económico (OCDE) la Educación Financiera, es el mecanismo mediante el cual, consumidores como instituciones financieras adquieren conocimiento de los diversos productos financieros, sus riesgos y beneficios, y a través de esta información o instrucción, desarrollan habilidades que les permite una mejor toma de decisiones, en la adquisición de estos productos o servicios. (OCDE, 2005)

En México la Educación Financiera está establecida en la Estrategia Nacional de Educación Financiera, como un conjunto de acciones para que la población adquiera aptitudes, habilidades y conocimientos y herramientas necesarias que les otorgue la oportunidad administrar y planear sus finanzas personales, así como analizar, evaluar y tomar decisiones de manera óptima y eficiente los productos y servicios que ofrece el sistema financiero en beneficio de sus intereses personales, familiares, laborales, profesionales, y de su negocio. Dicha educación es importante a nivel individual y social ya que contribuye al bienestar financiero y a la resiliencia de las personas y de las micro, pequeñas y medianas empresa, así como promueve a nivel nacional estabilidad económica e impulsa el crecimiento. (Consejo Nacional de Inclusion Financiera, 2018)

La Educación Financiera en México es implementada principalmente a nivel profesional (universitario) o por medio de cursos de capacitación, sin embargo los programas educativos de nivel básico escolar no cuentan con una materia diseñada específica para preparar a las nuevas generaciones para el uso eficiente de los recursos.
Durante sus estudios tomo alguna materia de educación financiera personal?

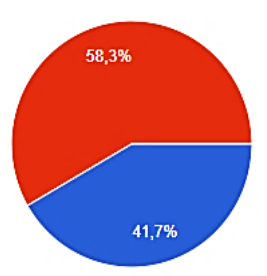

$$
\underset{\text { SI }}{\text { No }}
$$

Gráfico 1 Personas que mencionaron tener algún tipo de educación financiera personal durante su educación escolar

Fuente: elaboración propia

Considera que el uso de sus recursos financieros es eficiente? 24 respuestas

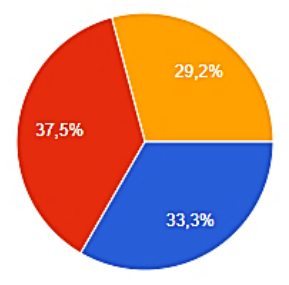

o $\mathrm{sl}$

No sabe

Grafico 2

Fuente: elaboración propia

Maneja alguna inversión o fondo de ahorro para el futuro? 24 respuestas
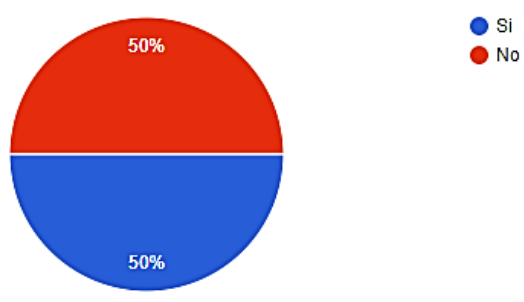

Grafico 3

Fuente: elaboración propia

\section{Metodología a desarrollar}

El presente estudio realizo una exhaustiva revisión de la literatura en cuanto a los contenidos de los programas académicos a nivel básico para primaria y secundaria, además se aplicó una prueba piloto una prueba para conocer el nivel de educación financiera de los adultos y saber si

\section{Resultados}

Los resultados de la prueba piloto proporciono información valiosa en educación financiera, dos aspectos que son preponderantes: 
En el primer aspecto permite contrastar los hallazgos encontrados en otras investigaciones y en Segundo lugar brinda un diagnóstico a nivel de competencias básicas en la persona encuestada. Derivado del análisis de la prueba se evidencio que de acuerdo a los resultados de la encuesta y el puntaje total obtenido en la prueba, no existen diferencias estadísticamente significativas entre los hombres y mujeres. Por lo menos en cuanto a educación financiera, sin embargo la que se obtiene es muy básica conociendo solamente banca de primer piso ya que todos los encuestados dan como observación que el porcentaje de educación financiera que se tiene entre hombres o mujeres es igual.

\section{Discusión}

Los resultados de esta investigación concuerdan con los de (Monica Vargas Prieto, 2014) en el que aplicaron un instrumento aplicado a 410 estudiantes determinaron que no existe diferencia entre el nivel de educación financiera entre hombres y mujeres. En México los resultados obtenidos por Racanello (2014) muestran que existe el $85 \%$ de la población no cuentan con un servicio financiero formal debido a la falta de conocimiento de los servicios ofertados por instituciones financieras (Raccanello \& Herrera Guzmán, Educación e inclusión financiera, 2014)

\section{Agradecimiento}

Agradezco a la Universidad Tecnológica de Altamira por el apoyo que se otorgó para llevar a cabo esta prueba piloto y medir la educación financiera de la comunidad escolar tanto como Docente así como alumnos.

\section{Conclusiones}

Los datos obtenidos por la diversa literatura analizada muestran la importancia y relevancia de la educación financiera desde la edad escolar básica ya que es una herramienta fundamental para el manejo óptimo de los recursos financieros de los hogares lo que tiene un impacto positivo en el bienestar de las familias y en la estabilidad económica del país. Este estudio permite desarrollar un instrumento para la evaluación financiera de las personas y observar el impacto en el empoderamiento de los grupos vulnerables.

\section{Referencias}

Consejo Nacional de Inclusion Financiera. (2018). REPORTE NACIONAL DE EDUCACION FINANCIERA. Mexico: CNBV.

Monica Vargas Prieto, y. B. (2014). Diseño y análisis psicométrico de un instrumento que evalua la competencia vasicas en Economia y Finanzas: una contribucion a la educacion para el consumo. Universitas Psychologica, 13791393.

OCDE. (2005). Recommendation on Principles and Good Practices for Financial. OCDE.

Raccanello, K., \& Herrera Guzmán, E. (2014). Educación e inclusión financiera. Revista Latinoamericana de Estudios Educativos, 119141.

Raccanello, K., \& Herrera Guzmán, E. (2014). Educación e inclusión financiera . Revista Latinoamericana de Estudios Educativos (México), 119-141.

Sarmiento, A. d. (2016). Análisis de la tenencia de productos financieros: evidencia para contribuir a la inclusión financiera en Bolivia . Red de Investigadores de bancos Centrales, 1752.

Tribín-Uribe, A. M.-B.-B.-G. (2016). Ahorro de los hogares de ingresos medios y bajos de las zonas urbana y rural en Colombia. Borradores de Economia, 960. 